\title{
Modulation of persistent photoconductivity by electric-field-controlled strain in thin films of $\mathrm{La}_{0.39} \mathrm{Pr}_{0.24} \mathrm{Ca}_{0.37} \mathrm{MnO}_{3}$
}

\author{
J. F. Wang, Y. C. Jiang, Z. P. Wu, and J. Gao ${ }^{\text {a) }}$ \\ Department of Physics, The University of Hong Kong, Pokfulam Road, Hong Kong, Hong Kong
}

(Received 2 September 2012; accepted 12 February 2013; published online 22 February 2013)

\begin{abstract}
Thin films of $\mathrm{La}_{0.39} \mathrm{Pr}_{0.24} \mathrm{Ca}_{0.37} \mathrm{MnO}_{3}$ (LPCMO) were epitaxially grown on $0.7 \mathrm{PbMg}_{1 / 3} \mathrm{Nb}_{2 / 3} \mathrm{O}_{3^{-}}$ $0.3 \mathrm{PbTiO}_{3}$ (PMN-PT) substrates. These LPCMO films exhibited low-temperature persistent photoconductivity (PPC) when illuminated by a visible light of $532 \mathrm{~nm}$. Not only the resistance but also PPC were modulated by electric fields across PMN-PT. Detailed analysis and in situ x-ray diffraction indicate that such modulations were mainly due to the change of lattice deformation by applied electric fields via converse piezoelectric effect. It was also found that light and strain influence the phase competition in different ways. (C) 2013 American Institute of Physics.
\end{abstract}

[http://dx.doi.org/10.1063/1.4793534]

Perovskite manganites are a family of materials with strong correlation. ${ }^{1-3}$ These materials exhibit many tantalizing properties, e.g., colossal magnetoresistance (CMR) effect. ${ }^{4,5}$ In the early studies, magnetoresistance effect in manganites was explained by double-exchange interaction, which results in simultaneous magnetism and conduction. When a magnetic field is applied, the core spins are aligned and the conduction is thus promoted. Later, it was pointed out that double exchange alone cannot explain the huge magnitude of CMR. Further studies suggested that CMR arises from the intimate coupling between different degrees of freedom and the intricate balance between diverse electronic ground states with close energy. One consequence of such coupling and balance is that the properties of manganites can be affected by various external perturbations, including magnetic field, electric field/current, light and pressure/strain. This feature is not only favorable for practical application but also useful for the exploration of underlying physics.

The studies on photo-induced effects are quite active but not as extensive as those on CMR effects. ${ }^{6-23}$ The origin of these effects has not been well-understood yet. Miyano et al. demonstrated a photo-induced transition from chargeordered state to metallic state in an electric-field biased $\mathrm{Pr}_{0.7} \mathrm{Ca}_{0.3} \mathrm{MnO}_{3}$ single crystal. ${ }^{6}$ The induced state is not stable after the removal of the electric field and the visible light. Further studies indicated that the photo-induced change without the assisting electric field can be achieved and the changes can be persistent. ${ }^{7}$ Besides resistance, other parameters, such as magnetization and reflectivity, also change upon the irradiation. ${ }^{7-11}$ Different from magnetic field, light illumination cannot only destroy charge-ordered state but also destabilize ferromagnetic metallic states. ${ }^{17,19}$

Since the estimated penetration depth of visible lights is several hundred nanometers, manganites in the form of thin film can make the whole parts illuminated. On the other hand, the properties of thin films can be different from those of bulk. The temperature dependence of resistance usually differs for films with identical thickness on different

\footnotetext{
${ }^{\text {a) }}$ Author to whom correspondence should be addressed. Electronic mail: jugao@hku.hk.
}

substrate and evolves with film thickness on the same substrate. The variation was attributed to strain effects. As strain can influence both the double exchange interaction and Jahn-Teller distortion, it should also play an important role in photo-induced changes. One concern for comparing different samples is that not only strain but also other factors, such as oxygen nonstoichiometry, may contribute to the variance significantly. ${ }^{24,25}$ Using piezoelectric substrates is a recently developed method and seems promising for the identification of strain effects. ${ }^{22,26-33}$ In this work, we employ a $0.7 \mathrm{PbMg}_{1 / 3} \mathrm{Nb}_{2 / 3} \mathrm{O}_{3}-0.3 \mathrm{PbTiO}_{3}$ (PMN-PT) substrate to investigate the effects of strain on the persistent photoconductivity in thin films of $\mathrm{La}_{0.39} \mathrm{Pr}_{0.24} \mathrm{Ca}_{0.37} \mathrm{MnO}_{3}$ (LPCMO). Our results demonstrate that the photo-induced effects can be altered significantly through electric-fieldcontrolled lattice deformation.

Thin films of LPCMO with thicknesses of $90 \mathrm{~nm}$ (denoted as LPCMO90) and $120 \mathrm{~nm}$ (denoted as LPCMO120) were grown on $(001)$ oriented $3 \times 5 \times 0.5 \mathrm{~mm}^{3}$ PMN-PT substrates using pulsed laser deposition technique. The film thickness was controlled by deposition time. The grown temperature was $650^{\circ} \mathrm{C}$, as monitored by k-type thermal couple. During deposition, oxygen pressure was maintained at $70 \mathrm{~Pa}$. Subsequent to film growth, the post-annealing treatment was made in situ at deposition temperature in a $10^{5}$-Pa oxygen atmosphere. Then, the samples were cooled to room temperature slowly. The silver electrodes on the surface of LPCMO films and the back of PMN-PT substrates were deposited using thermal evaporation. A Keithley 6487 voltage source was used to supply the dc voltage across PMN-PT. To limit the possibility of substrate crack as a result of polarization reversal, most measurements were done in a unipolar manner $(0 \mathrm{~V}<\mathrm{V}<500 \mathrm{~V}, 0 \mathrm{kV} / \mathrm{cm}<\mathrm{E}<10 \mathrm{kV} / \mathrm{cm})$. A semiconductor laser diode (wavelength $=532 \mathrm{~nm}$ ) with a power density of $2 \mathrm{~mW} / \mathrm{cm}^{2}$ was used as the light source.

The structural properties of LPCMO were characterized by X-ray diffraction (XRD). As can be seen from the XRD spectra in Fig. 1, LPCMO films were grown epitaxially with c-axis normal to the PMN-PT surface. There are no secondary phases visible. Typical rocking curves show a full width at half-maximum of $\sim 0.3^{\circ}$, indicating good crystallinity. 


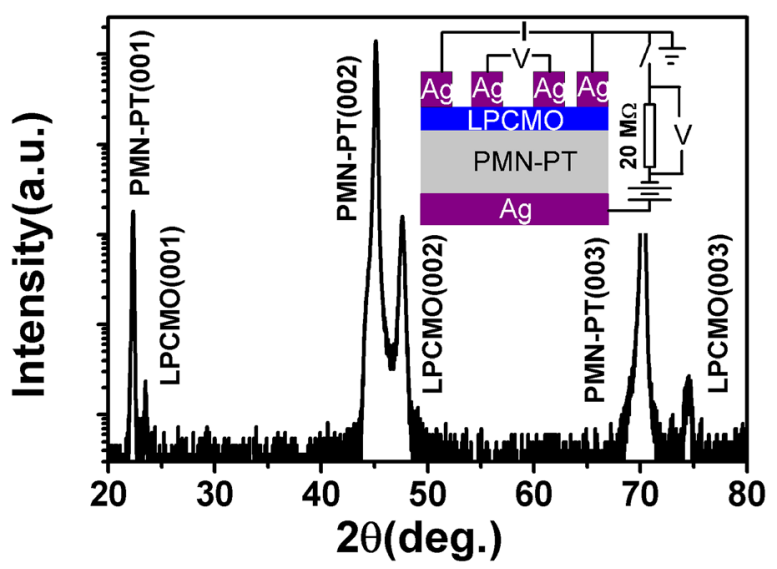

FIG. 1. A typical X-ray diffraction pattern for LPCMO film $(t=90 \mathrm{~nm})$ on PMN-PT. Shown in the inset is a schematic diagram of measurement circuit.

The transport properties of LPCMO films were measured in a closed-cycle cryostat using the circuit shown in the inset of Fig. 1. The polarity shown in the schematic diagram is defined as positive. The poling was made by applying an electric field of $10 \mathrm{kV} / \mathrm{cm}$ to PMN-PT (normal to the surface) at $325 \mathrm{~K}$ for $1 \mathrm{~h}$. After PMN-PT had been poled, temperature dependences of resistance were measured with different positive (the same polarity as the poling field) voltages, as displayed in Figs. 2(a) and 2(b). The samples exhibit an insulator-metal (IM) transition on cooling and a metalinsulator (MI) transition upon heating. The hysteresis in $\mathrm{R}$ vs $\mathrm{T}$ curves is a typical feature of ( $\mathrm{LaPrCa}) \mathrm{MnO}_{3}$ system. Near such a hysteresis, phase separation occurs and these phases are in competition. The applied electric fields effectively tuned the resistance in the whole temperature range. As voltage increased, resistance was reduced and temperatures of both IM $\left(\mathrm{T}_{\mathrm{IM}}\right)$ and $\mathrm{MI}\left(\mathrm{T}_{\mathrm{MI}}\right)$ transitions moved toward higher temperatures continuously. The temperature dependences of resistance modulation under an electric field of $10 \mathrm{kV} / \mathrm{cm}(\mathrm{V}=500 \mathrm{~V})$, $|\Delta \mathrm{R}|_{10 \mathrm{kV} / \mathrm{cm}} / \mathrm{R}=|[\mathrm{R}(10 \mathrm{kV} / \mathrm{cm})-\mathrm{R}(0)]| / \mathrm{R}(0)$ are exhibited in Figs. 2(c) and 2(d), respectively. $|\Delta R|_{10 \mathrm{kV} / \mathrm{cm}} / \mathrm{R}$ also shows a hysteresis between the cooling and warming processes. For

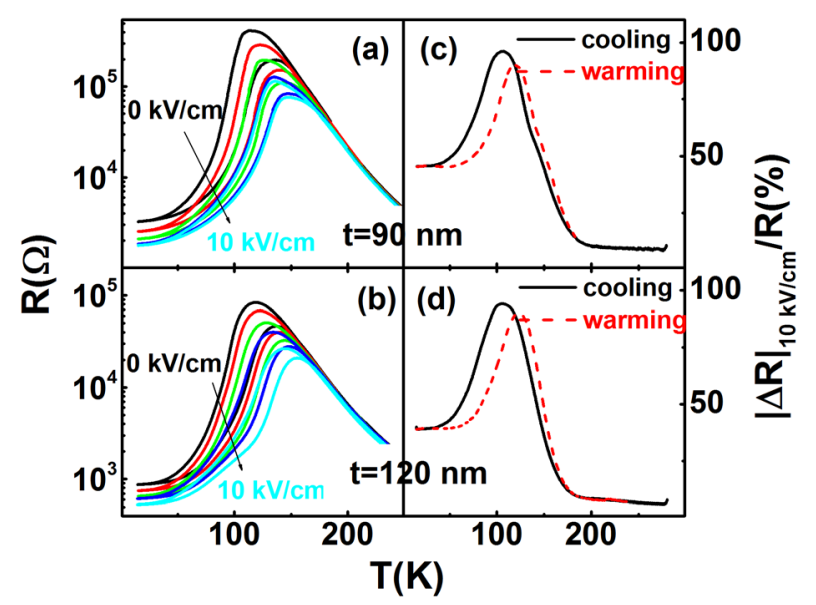

FIG. 2. (a) and (b) R(T) curves for LPCMO films on PMN-PT [(a) $t=90 \mathrm{~nm}$ and (b) $\mathrm{t}=120 \mathrm{~nm}$ ] under different bias voltages [from $500 \mathrm{~V}(10 \mathrm{kV} / \mathrm{cm})$ to $0 \mathrm{~V}(0 \mathrm{kV} / \mathrm{cm})$, the interval is $125 \mathrm{~V}(2.5 \mathrm{kV} / \mathrm{cm})]$. (c) and (d) Temperature dependences of resistance modulation, $|\Delta \mathrm{R}|_{10 \mathrm{kV} / \mathrm{cm}} / \mathrm{R}=\mid[\mathrm{R}(10 \mathrm{kV} / \mathrm{cm})$ $-\mathrm{R}(0)] \mid / \mathrm{R}(0)$ for LPCMO films $[(\mathrm{c}) \mathrm{t}=90 \mathrm{~nm}$ and $(\mathrm{d}) \mathrm{t}=120 \mathrm{~nm}]$. both processes, the maximum modulation is as large as $\sim 90 \%$, indicating an effective modulation of phase competition.

In our experiments, when the poled PMN-PT substrates was kept in a unipolar manner (for positively poled sample, $0 \mathrm{kV} / \mathrm{cm}=<\mathrm{E}=<10 \mathrm{kV} / \mathrm{cm}$ ), the leakage currents did not exceed $25 \mathrm{nA}$ (usually below $10 \mathrm{nA}$ ), indicating that the level of carrier injection is very low. It is apparent that the magnetic field generated by the leakage current is negligible. Such a magnitude of leakage currents is much less than the applied current for recording resistance $(\mathrm{I}=10 \mu \mathrm{A})$ and thus have trifling influence on measuring transport properties. This is verified for $\mathrm{E}=10 \mathrm{kV} / \mathrm{cm}$ by comparing the results measured with bias currents of $+10 \mu \mathrm{A}$ and $-10 \mu \mathrm{A}$ as well as the differential resistance (sweeping current from $-10 \mu \mathrm{A}$ to $10 \mu \mathrm{A})$. Generally, applying an electric field across PMN-PT can result in both a ferroelectric field effect and electromechanical effects. It has been shown that due to the high carrier density $\left(\sim 10^{22} / \mathrm{cm}^{3}\right),{ }^{2}$ manganites have a very short screening length $(<1 \mathrm{~nm}) .{ }^{34}$ When the applied electric field decreases from $10 \mathrm{kV} / \mathrm{cm}$ to $0 \mathrm{kV} / \mathrm{cm}$, the relative change in carrier density $\Delta \mathrm{n} / \mathrm{n}$ can be estimated from $\Delta \mathrm{n} / \mathrm{n}=\Delta \mathrm{P} / \mathrm{nt}$, where $\Delta \mathrm{P}[=\mathrm{P}(10 \mathrm{kV} / \mathrm{cm})-\mathrm{P}(0 \mathrm{kV} / \mathrm{cm})]$ is the change of polarization, $n$ is the carrier density in LPCMO, and $t$ is the thickness of LPCMO. With the parameters $\Delta \mathrm{P}=5 \mu \mathrm{C} / \mathrm{cm}^{2},{ }^{29} \mathrm{n}=10^{22} / \mathrm{cm}^{3}$, and $\mathrm{t}=100 \mathrm{~nm}, \Delta \mathrm{n} / \mathrm{n}$ is estimated to be $\sim 0.03 \%$. Such a tiny alteration in $\mathrm{n}$ cannot be the major reason for modulation of resistance, which is $\sim 10 \%$ at room temperature and larger at lower temperatures. To further confirm the minor role of surface charge, we applied an electric field of $-10 \mathrm{kV} / \mathrm{cm}$ (larger than the coercive field of PMN-PT, ${ }^{29} \mathrm{E}_{\mathrm{coer}} \sim 2 \mathrm{kV} / \mathrm{cm}$ ) to PMNPT at $325 \mathrm{~K}$. It is found that the temperature dependence of resistance under an electric field of $-10 \mathrm{kV} / \mathrm{cm}$ almost coincides with that under $\mathrm{E}=10 \mathrm{kV} / \mathrm{cm}$. Furthermore, $[\mathrm{R}(10 \mathrm{kV} / \mathrm{cm}) / \mathrm{R}(0)-1]_{10 \mathrm{kV} / \mathrm{cm} \rightarrow 0}$ is essentially the same as $[R(-10 \mathrm{kV} / \mathrm{cm}) / \mathrm{R}(0)-1]_{-10 \mathrm{kV} / \mathrm{cm} \rightarrow 0}$. Therefore, ferroelectric field effect should have negligible impacts on the resistance modulation. The systematic shifts of XRD reflection peaks upon the applied electric fields [see Figs. 3(a) and 3(b)] demonstrate that the electric field successfully controlled lattice distortion of PMN-PT and LPCMO. In accordance with previous reports using PMN-PT with similar compositions under electric fields of comparable magnitude, ${ }^{27,29,31}$ both $\Delta \mathrm{c} / \mathrm{c}_{\mathrm{PMN}-\mathrm{PT}}$ and $\Delta \mathrm{c} / \mathrm{c}_{\mathrm{LPCMO}}$ were found to change linearly with applied electric field [see Figs. 3(c) and $3(d)$, indicating a piezoelectric nature of the electric fieldinduced strain. Although there is a slight discrepancy in the out-of-plane lattice constant in PMN-PT, an electric field of $10 \mathrm{kV} / \mathrm{cm}$ enlarged c by $\sim 0.20 \%$ for both substrates. ${ }^{27,29,31}$ Consequently, the out-of-plane lattice strain decreases from $\sim 0.83 \%$ to $\sim 0.70 \%$ and from $\sim 0.74 \%$ to $\sim 0.62 \%$ for LPCMO90 and LPCMO120, respectively.

The light illuminated the sample through the optical window of the closed-cycle cryostat. The laser spot fully covered the gap between the inner two electrodes (see the inset of Fig. 1). Figure 4(a) shows the time evolution of resistance of the LPCMO90 film when the light was switched on and off at $15 \mathrm{~K}$. When the sample was kept in dark, the resistance was essentially stable. At the beginning of the illumination, the resistance dropped sharply. Then the decrease 

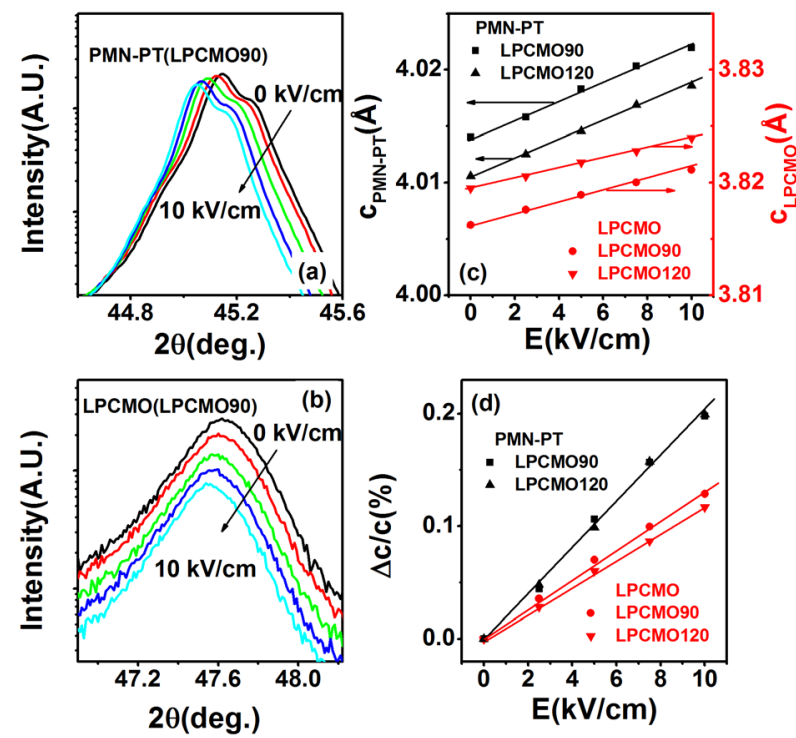

FIG. 3. (a) and (b) In situ XRD patterns in the vicinity of (002) reflections for PMN-PT substrate (LPCMO90 sample) (a) and LPCMO90 film (b) under different electric fields [from $500 \mathrm{~V}(10 \mathrm{kV} / \mathrm{cm})$ to $0 \mathrm{~V}(0 \mathrm{kV} / \mathrm{cm})$, the interval is $125 \mathrm{~V}(2.5 \mathrm{kV} / \mathrm{cm})]$. (c)and (d) Electric field dependence of out-ofplane lattice constant $\mathrm{c}$ (c) and its relative change $\Delta \mathrm{c} / \mathrm{c}$ (d) of both films and substrates for LPCMO90 and LPCMO120.

of resistance became slowly with further irradiation. The light was turned off after $1-\mathrm{h}$ irradiation. It is interesting to note that the resistance did not recover to the initial value. This phenomenon characterizes a persistent photoconductivity (PPC) effect. ${ }^{7,8,12,23}$ Instead, there is a slight drop [see the inset of Fig. 4(a)]. Such a change is reversible. If the light is switched on again, the resistance will increase to the value just before the light is removed. This transient change of

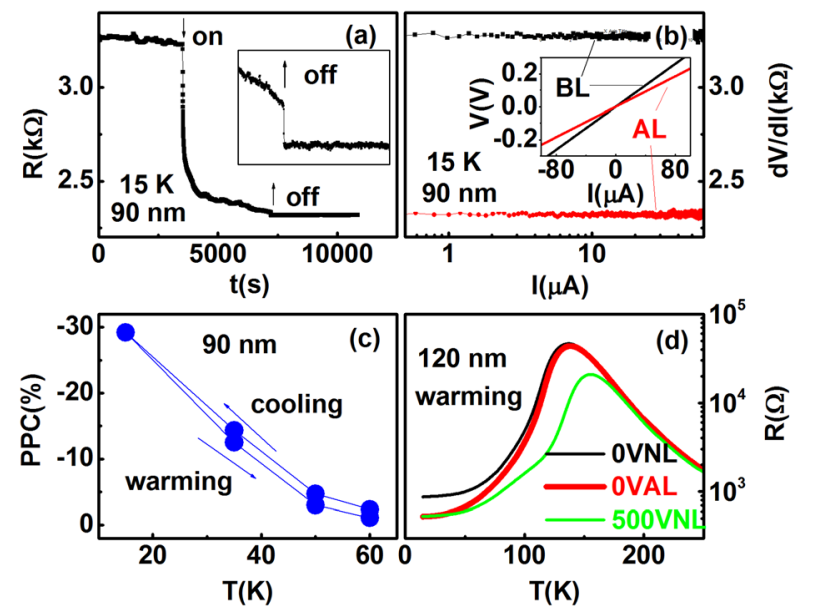

FIG. 4. Resistance versus illumination time for LPCMO on PMN-PT $(\mathrm{t}=90 \mathrm{~nm}$, voltage across $\mathrm{PMN}-\mathrm{PT}=0 \mathrm{~V}, \mathrm{~T}=15 \mathrm{~K}, \quad \mathrm{I}=10 \mu \mathrm{A}) . \quad(\mathrm{b})$ Differential Resistance as a function of current and voltage vs current (inset) curves before (dark) and after (red) light illumination for LPCMO on PMNPT $(\mathrm{t}=90 \mathrm{~nm}$, voltage across PMN-PT $=0 \mathrm{~V}, \mathrm{~T}=15 \mathrm{~K})$. (c) Temperature dependence of PPC (illumination duration $=1 \mathrm{~h}$ ) for LPCMO on PMN-PT $\left(\mathrm{t}=90 \mathrm{~nm}, \mathrm{~V}_{\mathrm{PMN}-\mathrm{PT}}=0 \mathrm{~V}\right)$. (d) Comparison of $\mathrm{R}(\mathrm{T})$ curves during the warming process measured in dark $(\mathrm{t}=120 \mathrm{~nm}$ and $\mathrm{I}=10 \mu \mathrm{A})$. Two of them were measured with $\mathrm{V}_{\mathrm{PMN}-\mathrm{PT}}=0 \mathrm{~V}$ : the thin lines $(0 \mathrm{VNL})$ are recorded without light illumination and the thick red line (OVAL) is measured after illumination for $1 \mathrm{~h}$ at $15 \mathrm{~K}$. The third (shown with green thin line, denoted as $500 \mathrm{VNL})$ is the $\mathrm{R}(\mathrm{T})$ curve under $500 \mathrm{~V}$ bias $\left(\mathrm{V}_{\mathrm{PMN}-\mathrm{PT}}=500 \mathrm{~V}\right.$, $\mathrm{E}=10 \mathrm{kV} / \mathrm{cm})$. resistance is probably caused by heating induced by laser irradiation. As the temperature coefficient $(\mathrm{dR} / \mathrm{dT})$ below $\mathrm{T}_{\mathrm{MI}} / \mathrm{T}_{\mathrm{IM}}$ is positive, the heating effect caused by laser will increase the resistance and the resistance will drop after the light is switched off. ${ }^{15,21}$ The observed PPC effect is independent of the polarity and magnitude of bias current [see Fig. 4(b)]. This is different from the PPC induced by currents in $\mathrm{Pr}_{0.7} \mathrm{Sr}_{0.3} \mathrm{MnO}_{3}{ }^{20}$ The clearly linear current-voltage curves also indicate that this effect should be irrelevant to interfacial effects between electrodes and LPCMO.

To further understand the PPC effects, similar measurements were carried out at different temperatures. Figure 4(c) shows the temperature dependence of PPC for LPCMO90. Here, the PPC is defined as $\mathrm{PPC}=100 \times[\mathrm{R}(\mathrm{AL})-\mathrm{R}(\mathrm{PL})] /$ $\mathrm{R}(\mathrm{PL})$, where $\mathrm{R}(\mathrm{PL})$ and $\mathrm{R}(\mathrm{AL})$ are the resistance prior to illumination and after 1-h illumination (in dark, the light is switched off), respectively. Prior to each measurement, the sample was heated to room temperature to quench any residual PPC effects. ${ }^{20,21,23}$ There is also tiny hysteresis in the temperature dependence of PPC. At one selected temperature, the magnitude of PPC during cooling process (cooling the sample in the dark from $300 \mathrm{~K}$ to the selected temperature) is slightly larger than that upon warming (first cooling the sample from $300 \mathrm{~K}$ to $15 \mathrm{~K}$ and then heating it to the target temperature). The general trend is that the lower the temperature, the larger the magnitude of PPC. Above $60 \mathrm{~K}$, PPC is negligible. This is in accordance with previous reports. ${ }^{9,23}$ As shown in Figs. 2(c) and 2(d), the maximum resistance modulations induced by lattice strain appear at temperatures slightly lower than $\mathrm{T}_{\mathrm{IM}}\left(\mathrm{T}_{\mathrm{MI}}\right)$, where strong phase competition between metallic and insulating phases occurs. On contrary, PPC does not peak in the temperature region with strongest phase competition. PPC appeared at much lower temperatures and exhibited a monotonous increase with the decreases of temperature [see Fig. 4(c)]. However, this feature cannot exclude phase competition from essential ingredients of PPC. For more metallic $\mathrm{Pr}_{0.7} \mathrm{Sr}_{0.3} \mathrm{MnO}_{3}$ and more insulating $\mathrm{Sm}_{0.55} \mathrm{Sr}_{0.45} \mathrm{MnO}_{3},{ }^{20,21}$ no PPC effects were observed. Thus, phase competition should also be important for PPC. Different temperature dependences of PPC and $|\Delta \mathrm{R}|_{10 \mathrm{kV} / \mathrm{cm}} / \mathrm{R}$ imply that light irradiation and lattice strain affect manganites in different manners.

It is noted that the low-resistance state after light illumination is different from that induced by lattice strain. As can be seen from Fig. 4(d), for LPCMO120 at $15 \mathrm{~K}$, the resistance with no voltage across PMN-PT after 1-h light irradiation (denoted as $0 \mathrm{VAL}$ ) is close to that with $\mathrm{V}_{\mathrm{PMN}-\mathrm{PT}}$ $=500 \mathrm{~V}(\mathrm{E}=10 \mathrm{kV} / \mathrm{cm})$ without illumination (denoted as $500 \mathrm{VNL}$ ). One might expect that these two states (0VAL and 500VNL) should have very similar temperature dependences of resistance while warming. However, as displayed in Fig. 4(d), it turns out that for these two states, both temperature of MI transition and the magnitude of resistance near MI are different. Actually, it can be seen that for $\mathrm{V}_{\mathrm{PMN}-\mathrm{PT}}=0 \mathrm{~V}$, the temperature of MI transition and the magnitude of resistance near MI transition are not influenced by whether the sample was illuminated at $15 \mathrm{~K}$. The underlying reason should be that PPC was quenched by raising temperatures. This also indicates that photo irradiation and strain affect the LPCMO in different ways. 


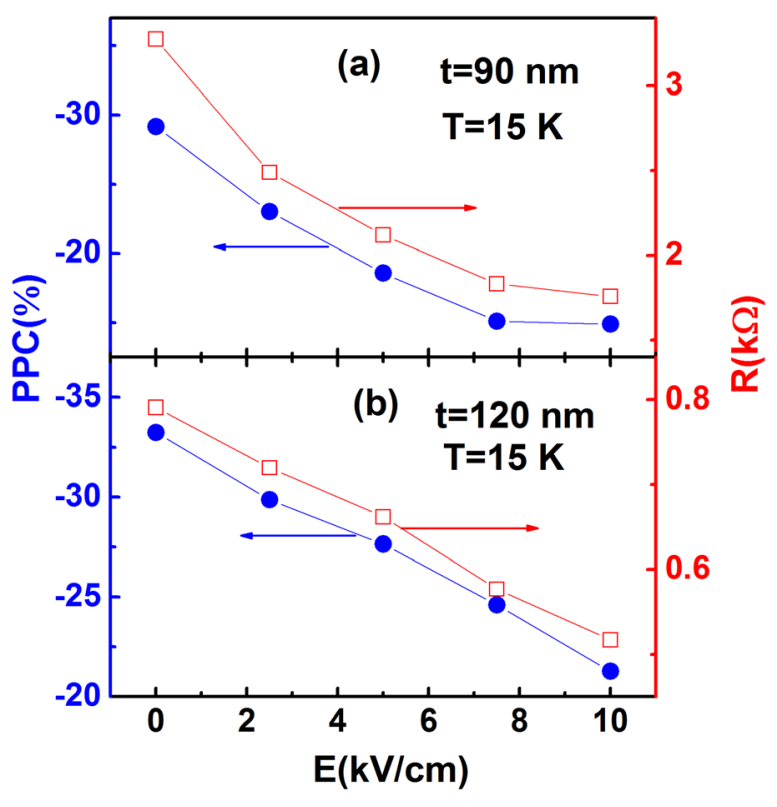

FIG. 5. PPC (illumination duration $=1 \mathrm{~h}$ ) at $15 \mathrm{~K}$ for LPCMO films on PMNPT [(a) $\mathrm{t}=90 \mathrm{~nm}$ and (b) $\mathrm{t}=120 \mathrm{~nm}$ ] under different bias voltages [from $500 \mathrm{~V}(10 \mathrm{kV} / \mathrm{cm})$ to $0 \mathrm{~V}(0 \mathrm{kV} / \mathrm{cm})$, and the interval is $125 \mathrm{~V}(2.5 \mathrm{kV} / \mathrm{cm})]$.

To see how the strain influences the PPC effects, the photo responses of resistance at $15 \mathrm{~K}$ were measured with different $\mathrm{V}_{\text {PMN-PT }}$. The results are summarized in Fig. 5. For both samples, the increasing positive voltages across PMN$\mathrm{PT}$ resulted in the decreasing resistance at $15 \mathrm{~K}$. The electric field dependence of resistance is not exactly linear. ${ }^{33}$ It is interesting to note that although the resistivity for LPCMO120 is lower than that of LPCMO90, the magnitude of PPC in LPCMO120 is slightly larger. Nevertheless, for both samples, the magnitude decreases with the increase of bias voltage. Applying an electric field of $10 \mathrm{kV} / \mathrm{cm}$ across PMN-PT caused a near 50\% drop in PPC. The observed PPC and its dependence upon applied electric field cannot be related to carrier injection from PMN-PT substrates. As the photon energy of incident light $(\lambda=532 \mathrm{~nm}, \mathrm{~h} \nu=2.33 \mathrm{eV})$ is smaller than the band gap of PMN-PT $\left(\mathrm{E}_{\mathrm{g}} \sim 3.2 \mathrm{eV}\right),{ }^{35}$ the incident light cannot generate electron-hole pairs in $\mathrm{PMN}$ PT. For LPCMO90 under an electric field of $-10 \mathrm{kV} / \mathrm{cm}$, we also measured PPC at $15 \mathrm{~K}$, which turned out to be very close to that with $\mathrm{E}=10 \mathrm{kV} / \mathrm{cm}$ and $\mathrm{T}=15 \mathrm{~K}$. This signifies that there is negligible injection of photo generated carrier. The modulation of PPC by the applied electric field should originate from the change of lattice distortion due to converse piezoelectric effect. As seen from Fig. 3, the electric field applied to PMN-PT reduced the out-of-plane compressing strain in PLCMO, which would partially relieve the inplane stretching in LPCMO. As a result of enhanced double exchange and suppressed Jahn-Teller distortion, LPCMO films become more metallic and PPC was suppressed.

In summary, we have deposited epitaxial LPCMO films on PMN-PT single crystals. It was observed that LPCMO films possess substantial PPC effects at low temperatures. The transport properties and PPC have been explored with varying voltages across PMN-PT. It was found that the electric field controlled lattice deformation can effectively shift the balance of phase competition and thus the resistance and PPC.

This work has been supported by a grant of the Research Grant Council of Hong Kong (Project No. HKU 702112P) and CRCG of HKU.

${ }^{1}$ E. Dagotto, T. Hotta and A. Moreo, Phys. Rep. 344, 1 (2001).

${ }^{2}$ M. B. Salamon and M. Jaime, Rev. Mod. Phys. 73, 583 (2001).

${ }^{3}$ Y. Tokura, Rep. Prog. Phys. 69, 797 (2006).

${ }^{4}$ R. von Helmolt, J. Wecker, B. Holzapfel, L. Schultz and K. Samwer, Phys. Rev. Lett. 71, 2331 (1993).

${ }^{5}$ S. Jin, T. H. Tiefel, M. McCormack, R. A. Fastnacht, R. Ramesh and L. H. Chen, Science 264, 413 (1994).

${ }^{6}$ K. Miyano, T. Tanaka, Y. Tomioka and Y. Tokura, Phys. Rev. Lett. 78, 4257 (1997).

${ }^{7}$ M. Baran, S. L. Gnatchenko, O. Y. Gorbenko, A. R. Kaul, R. Szymczak and H. Szymczak, Phys. Rev. B 60, 9244 (1999).

${ }^{8}$ H. Huhtinen, R. Laiho, E. Lähderanta, L. S. Vlasenko, M. P. Vlasenko and V. S. Zakhvalinskii, Phys. Rev. B 62, 11614 (2000).

${ }^{9}$ H. Oshima, M. Nakamura and K. Miyano, Phys. Rev. B 63, 075111 (2001).

${ }^{10}$ I. I. Smolyaninov, V. N. Smolyaninova, C. C. Davis, B. G. Kim, S. W. Cheong and R. L. Greene, Phys. Rev. Lett. 87, 127204 (2001).

${ }^{11}$ Y. Okimoto, Y. Ogimoto, M. Matsubara, Y. Tomioka, T. Kageyama, T. Hasegawa, H. Koinuma, M. Kawasaki and Y. Tokura, Appl. Phys. Lett. 80, 1031 (2002).

${ }^{12}$ R. Cauro, A. Gilabert, J. P. Contour, R. Lyonnet, M. G. Medici, J. C. Grenet, C. Leighton and I. K. Schuller, Phys. Rev. B 63, 174423 (2001).

${ }^{13}$ V. N. Smolyaninova, M. Rajeswari, R. Kennedy, M. Overby, S. E. Lofland, L. Z. Chen and R. L. Greene, Appl. Phys. Lett. 86, 071922 (2005).

${ }^{14}$ N. Takubo, Y. Ogimoto, M. Nakamura, H. Tamaru, M. Izumi and K. Miyano, Phys. Rev. Lett. 95, 017404 (2005).

${ }^{15}$ R. M. Kolagani, M. A. Overby, V. N. Smolyaninova, A. Davidson and D. E. Cox, Appl. Phys. Lett. 88, 052504 (2006).

${ }^{16}$ M. Matsubara, Y. Okimoto, T. Ogasawara, Y. Tomioka, H. Okamoto and Y. Tokura, Phys. Rev. Lett. 99, 207401 (2007).

${ }^{17}$ N. Takubo, I. Onishi, K. Takubo, T. Mizokawa and K. Miyano, Phys. Rev. Lett. 101, 177403 (2008).

${ }^{18}$ E. Beyreuther, A. Thiessen, S. Grafström, L. M. Eng, M. C. Dekker and K. Dörr, Phys. Rev. B 80, 075106 (2009).

${ }^{19}$ V. N. Smolyaninova, G. Yong, R. M. Kolagani, A. Biswas, H. K. Ermer, K. Wang and A. Piazza, Appl. Phys. Lett. 99, 222507 (2011).

${ }^{20}$ J. F. Wang and J. Gao, J. Appl. Phys. 109, 07D701 (2011).

${ }^{21}$ S. Chaudhuri, N. K. Pandey, S. Shrikant and R. C. Budhani, J. Phys.: Condens. Matter 22, 275502 (2010).

${ }^{22}$ E. J. Guo, J. Gao and H. B. Lu, Appl. Phys. Lett. 98, 081903 (2011).

${ }^{23}$ J. M. Dai, W. H. Song, S. G. Wang, S. L. Ye, K. Y. Wang, J. J. Du and Y. P. Sun, J. Appl. Phys. 90, 3118 (2001).

${ }^{24}$ P. Murugavel, J. H. Lee, J. G. Yoon, T. W. Noh, J. S. Chung, M. Heu and S. Yoon, Appl. Phys. Lett. 82, 1908 (2003).

${ }^{25}$ J. Dvorak, Y. U. Idzerda, S. B. Ogale, S. Shinde, T. Wu, T. Venkatesan, R. Godfrey and R. Ramesh, J. Appl. Phys. 97, 10C102 (2005).

${ }^{26}$ D. Dale, A. Fleet, J. D. Brock and Y. Suzuki, Appl. Phys. Lett. 82, 3725 (2003).

${ }^{27}$ C. Thiele, K. Dörr, S. Fahler, L. Schultz, D. C. Meyer, A. A. Levin and P. Paufler, Appl. Phys. Lett. 87, 262502 (2005).

${ }^{28}$ A. A. Levin, A. I. Pommrich, T. Weissbach, D. C. Meyer and O. BilaniZeneli, J. Appl. Phys. 103, 054102 (2008).

${ }^{29}$ R. K. Zheng, Y. Wang, H. L. W. Chan, C. L. Choy and H. S. Luo, Appl. Phys. Lett. 92, 082908 (2008).

${ }^{30}$ M. C. Dekker, A. D. Rata, K. Boldyreva, S. Oswald, L. Schultz and K. Dörr, Phys. Rev. B 80, 144402 (2009).

${ }^{31}$ M. D. Biegalski, K. Dörr, D. H. Kim and H. M. Christen, Appl. Phys. Lett. 96, 151905 (2010).

${ }^{32}$ A. Herklotz, J. D. Plumhof, A. Rastelli, O. G. Schmidt, L. Schultz and K. Dör, J. Appl. Phys. 108, 094101 (2010).

${ }^{33}$ J. F. Wang and J. Gao, Appl. Phys. Lett. 100, 131903 (2012).

${ }^{34}$ X. Hong, A. Posadas and C. H. Ahn, Appl. Phys. Lett. 86, 142501 (2005).

${ }^{35}$ C. He, F. Wang, D. Zhou, X. Zhao, D. Lin, H. Xu, T. He and H. Luo, J. Phys. D: Appl. Phys. 39, 4337 (2006). 